Supporting Information for

\title{
Anion-Radical Polymerization of Sulfur- and Selenium-Substituted N-Type Conjugated Polymers
}

\author{
Paniz Pahlavanlu, So Young An, Jenny R. Panchuk, Adam A. Pollit and Dwight S. Seferos* \\ Department of Chemistry, University of Toronto \\ 80 St. George Street, Toronto, Ontario, M5S 3H6, Canada \\ *Email:dwight.seferos@utoronto.ca
}

Table of Contents

1. Anion-Radical Polymerization Mechanism

2. Monomer and Polymer Scope

3. Synthesis and Characterization of Anion-Radical Monomers

4. Anion-Radical Polymerization of Oxygenated and Thionated Monomers

S11

5. Supplementary Computational Calculations

S15 


\section{Summary of Figures and Tables}

Scheme S1. Anion-radical polymerization mechanism, illustrating possible chain propagation and chain termination pathways.

Figure S1. Chemical structures and abbreviations of neutral monomers, anion-radical monomers, and polymers investigated in this work. $\quad$ S4

Table S1. Electronic properties of O-Th, O-Se, S1-Th, and S2-Th. $\quad$ S5

Figure S2. DFT-Calculated geometry optimizations of oxygenated and thionated neutral monomers, indicating amide and thioamide $\mathrm{C}-\mathrm{N}$ and $\mathrm{C}=\mathrm{O} / \mathrm{C}=\mathrm{S}$ bond lengths. S6

Figure S3. DFT-Calculated geometry optimizations (top) and electrostatic potential maps (bottom) of O-Th, S1-Th, and S2-Th. $\quad$ S6

Figure S4. Examples of possible resonance structures of the S2-Th anion-radical monomer. $\quad$ S7

Figure S5. EPR spectra of O-Th/Zn before and after addition of S2-Th. $\quad$ S7

Figure S6. Spectroelectrochemistry studies O-Th, O-Se, S1-Th, and S2-Th. $\quad$ S8

Figure S7. Normalized optical absorption spectra of anion-radical monomers compared to singly and doubly reduced states measured through spectroelectrochemical studies in Figure S4. $\quad$ S8 Figure S8. Time-resolved optical absorption spectra of a) O-Th/Zn, b) S1-Th/Zn, and c) S2-Th/Zn following exposure to ambient atmosphere to reform O-Th, S1-Th, and S2-Th, respectively. $\quad$ S9 Figure S9. ${ }^{1} \mathrm{H}$ NMR analysis of a) O-Th/Zn quenching product compared to b) diprotonated OTh, and c) O-Th, indicating reformation of O-Th following exposure to ambient atmosphere. S9 Figure S10. ${ }^{1} \mathrm{H}$ NMR analysis of a) S2-Th/Zn quenching product compared to b) diprotonated OTh, c) O-Th, d) S1-Th, and f) S2-Th, indicating reformation of S2-Th and partial oxidation to S1Th following exposure to ambient atmosphere.

S10

Table S2. Preliminary screening of $\mathrm{Ni}(\mathrm{dppe}) \mathrm{Cl}_{2}, \mathrm{Ni}(\mathrm{MesAn}) \mathrm{Br}_{2}$, and $\mathrm{Pd} / \mathrm{P}(t-\mathrm{Bu}) 3$ anion-radical $\begin{array}{ll}\text { polymerization catalysts. } & \mathbf{S 1 1}\end{array}$

Table S3. Summary of anion-radical polymerization reaction conditions and properties. $\quad$ S12 Figure S11. ${ }^{1} \mathrm{H}$ NMR analysis of O-Th and O-Se following treatment with excess $\mathrm{Ni}(\mathrm{dppp}) \mathrm{Cl}_{2}$ for 1 hour, compared to diprotonated and dibrominated compounds.

S13

Figure S12. Mass spectra of a) $\mathrm{S} 1-\mathrm{Th} / \mathrm{Zn}$ and b) $\mathrm{S} 2-\mathrm{Th} / \mathrm{Zn}$ after $\mathrm{Ni}(\mathrm{dppp}) \mathrm{Cl}_{2}$ treatment, indicating partial protonation and oxygen-substitution. $\quad$ S13

Figure S13. Representative ${ }^{1} \mathrm{H}$ NMR spectra of a) P(TNDIT) and b) P(SeNDISe). $\quad$ S14 Figure S14. DFT-calculated geometry optimizations and single point energy calculations of neutral and reduced $\mathrm{O}-\mathrm{Th}, \mathrm{O}-\mathrm{Se}, \mathrm{S} 1-\mathrm{Th}$, and S2-Th, indicating dihedral angles $\left(\theta_{\mathrm{DA}}\right)$ between NDI and $\mathrm{Th} / \mathrm{Se}$ units and relative energies between neutral and reduced monomers.

S15 


\section{Anion-Radical Polymerization Mechanism}

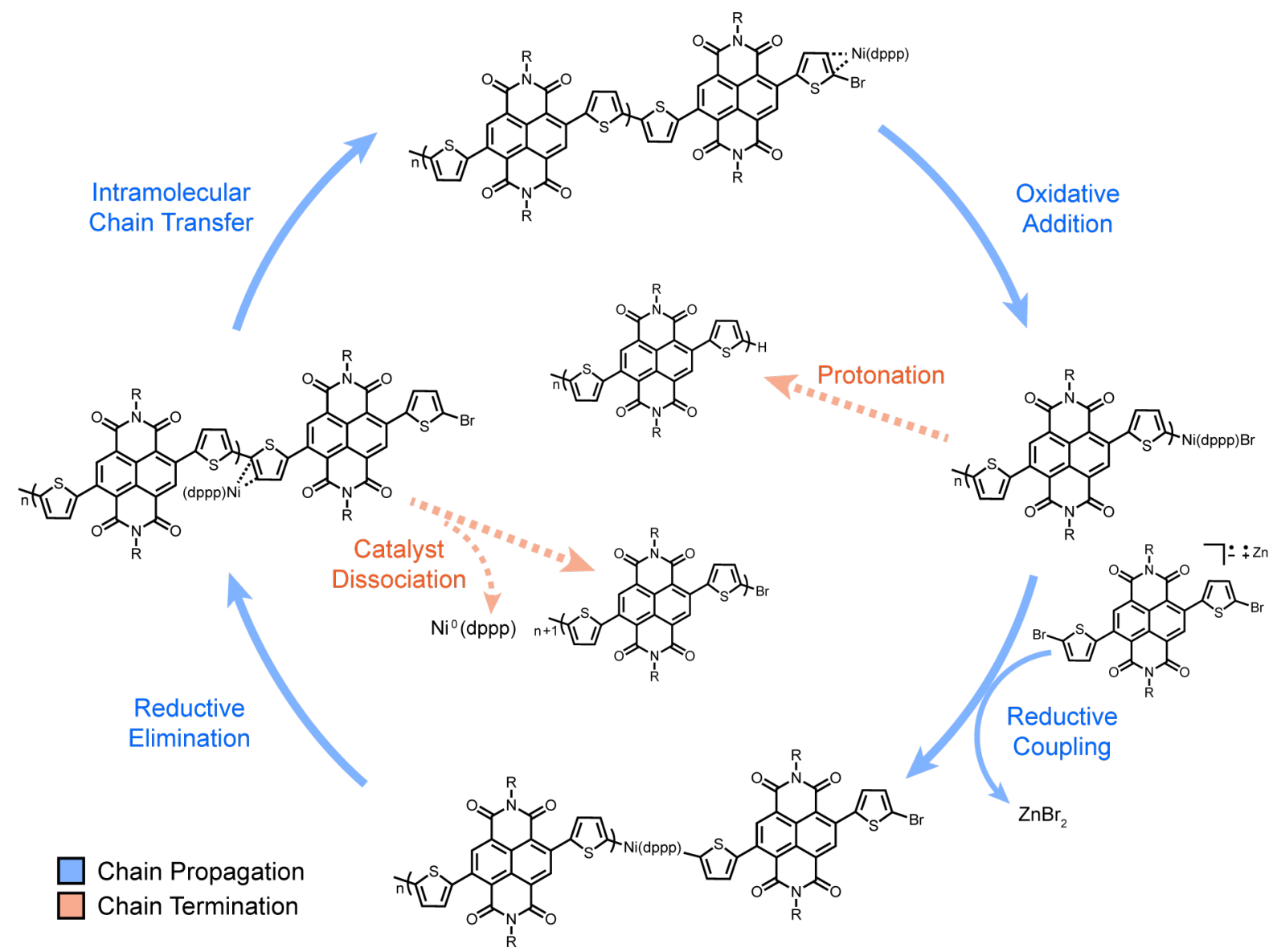

Scheme S1. Anion-radical polymerization mechanism, illustrating possible chain propagation and chain termination pathways. 


\section{Monomer and Polymer Scope}
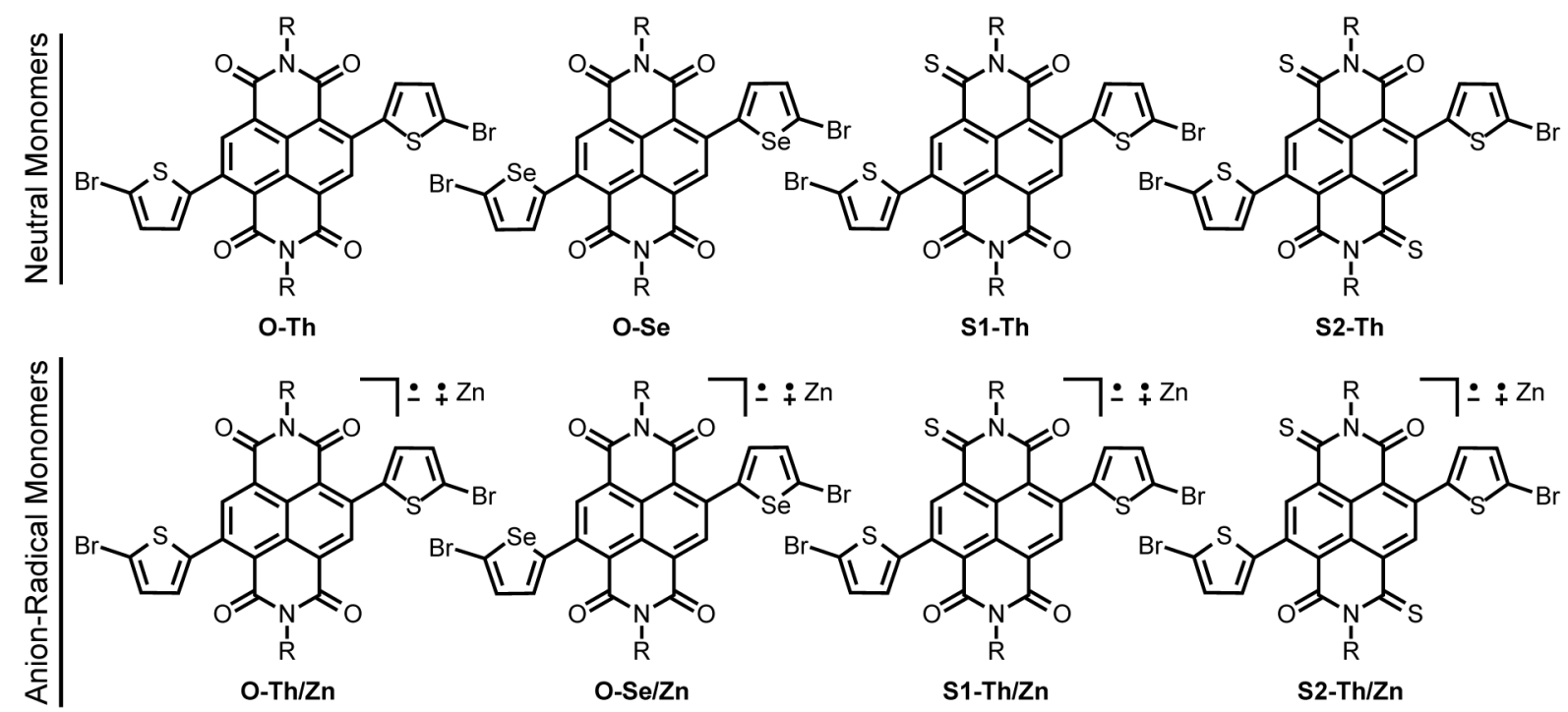

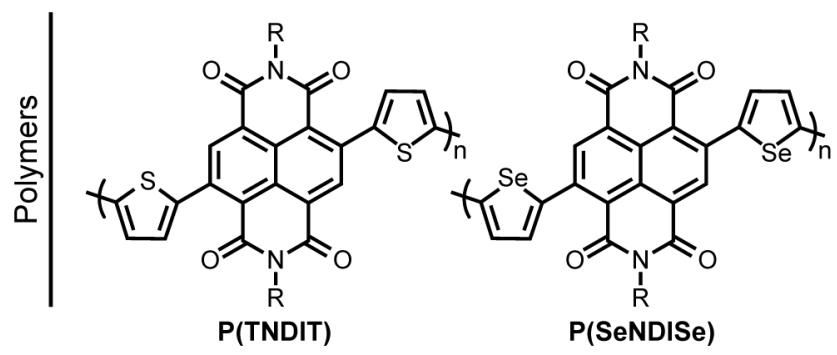

Figure S1. Chemical structures and abbreviations of neutral monomers, anion-radical monomers, and polymers investigated in this work. $\mathrm{R}=2$-octyldodecyl. 


\section{Synthesis and Characterization of Anion-Radical Monomers}

Table S1. Electronic properties of O-Th, O-Se, S1-Th, and S2-Th.

\begin{tabular}{|c|c|c|c|c|c|}
\hline & $\begin{array}{c}E_{\mathrm{R} 1,1 / 2}{ }^{\mathrm{a}} \\
(\mathrm{V})\end{array}$ & $\begin{array}{c}E_{\mathrm{R} 2,1 / 2}{ }^{\mathrm{a}} \\
(\mathrm{V})\end{array}$ & $\begin{array}{c}\mathrm{E}_{\mathrm{HOMO}} \mathrm{b}, \mathrm{c} \\
(\mathrm{eV})\end{array}$ & $\begin{array}{c}\mathrm{E}_{\text {LUMO }}{ }^{\mathrm{b}, \mathrm{d}} \\
(\mathrm{eV})\end{array}$ & g-factor ${ }^{\mathrm{e}}$ \\
\hline $\mathrm{O}-\mathrm{Th}$ & -1.01 & -1.47 & $\begin{array}{c}-6.16 \\
(-6.41)\end{array}$ & $\begin{array}{c}-4.09 \\
(-3.86)\end{array}$ & 2.0022 \\
\hline $\mathrm{O}-\mathrm{Se}$ & -1.04 & -1.47 & $\begin{array}{c}-6.10 \\
(-6.42)\end{array}$ & $\begin{array}{c}-4.06 \\
(-3.86)\end{array}$ & 2.0021 \\
\hline S1-Th & -0.96 & -1.35 & $\begin{array}{c}-6.05 \\
(-6.41)\end{array}$ & $\begin{array}{c}-4.14 \\
(-3.99)\end{array}$ & 2.0043 \\
\hline S2-Th & -0.77 & -1.09 & $\begin{array}{c}-6.15 \\
(-6.42)\end{array}$ & $\begin{array}{c}-4.33 \\
(-4.11)\end{array}$ & 2.0067 \\
\hline
\end{tabular}

${ }^{\mathrm{a}} E_{\mathrm{R} 1,1 / 2}$ and $E_{\mathrm{R} 2,1 / 2}$ are the first and second half-width reduction potentials, respectively, obtained by cyclic voltammetry referenced to the $\mathrm{Fc} / \mathrm{Fc}^{+}$redox couple. ${ }^{b} \mathrm{DFT}$ calculated orbital energies (B3LYP functional, 6-31G(d) basis set, converted from Hartrees to eV) are included in brackets below experimentally obtained energies. ${ }^{c}$ Experimentally obtained $\mathrm{E}_{\mathrm{HOMO}}=\mathrm{E}_{\mathrm{LUMO}}-\mathrm{E}_{\mathrm{g}, \mathrm{opt}}$, where $E_{g, o p t}$ is the optical energy gap calculated from the absorption onset by optical absorption spectroscopy. ${ }^{\mathrm{d}}$ Experimentally obtained $\mathrm{E}_{\mathrm{LUMO}}=-\left(\mathrm{E}_{\mathrm{R} 1,1 / 2}+5.1\right)$, where $\mathrm{E}_{\mathrm{R} 1,1 / 2}$ is the energy associated with the first half-width reduction potential. ${ }^{\mathrm{e}} \mathrm{g}$-factor of the corresponding zinc complex: O-Th/Zn, O-Se/Zn, S1-Th/Zn, or S2-Th/Zn. 

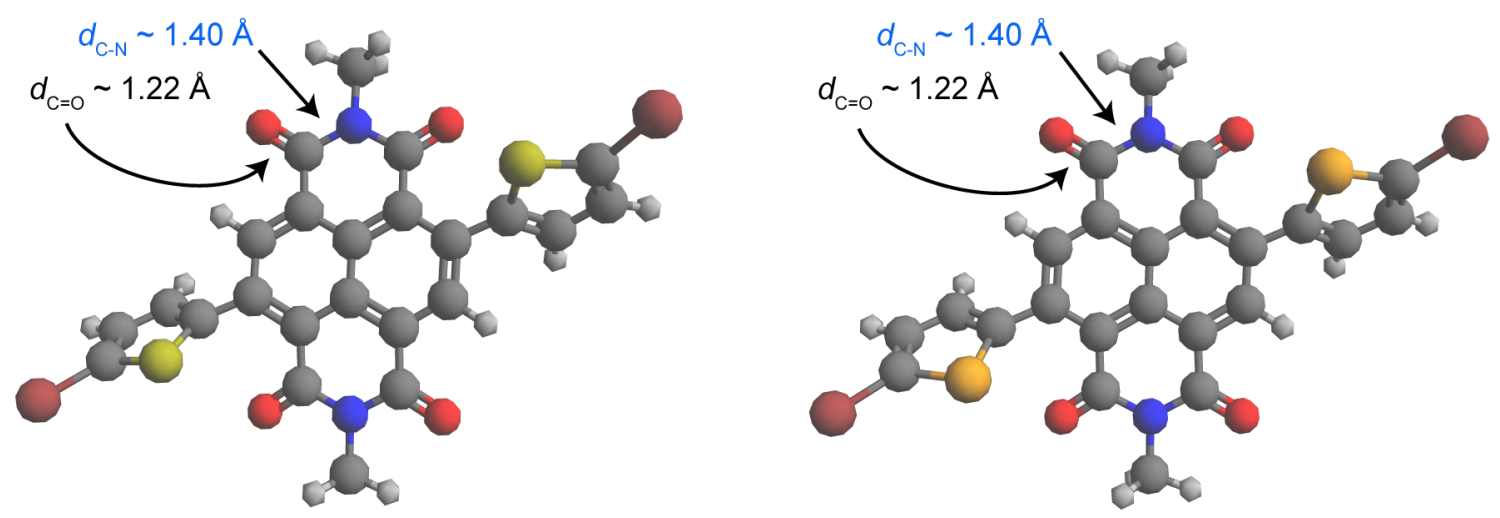

O-Th

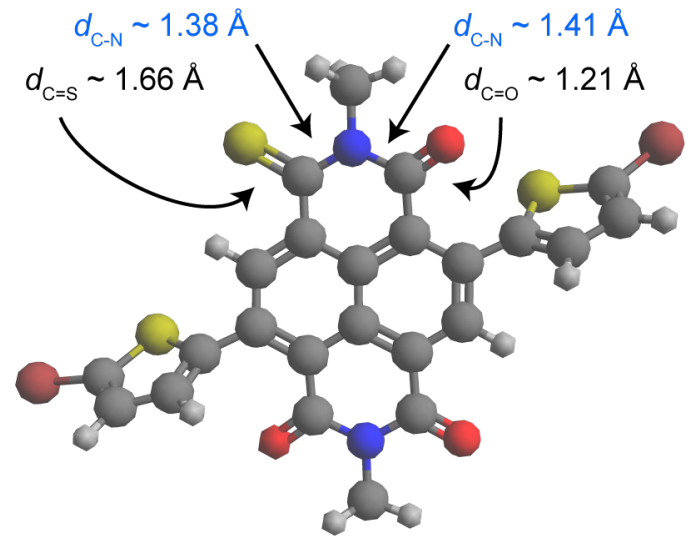

S1-Th

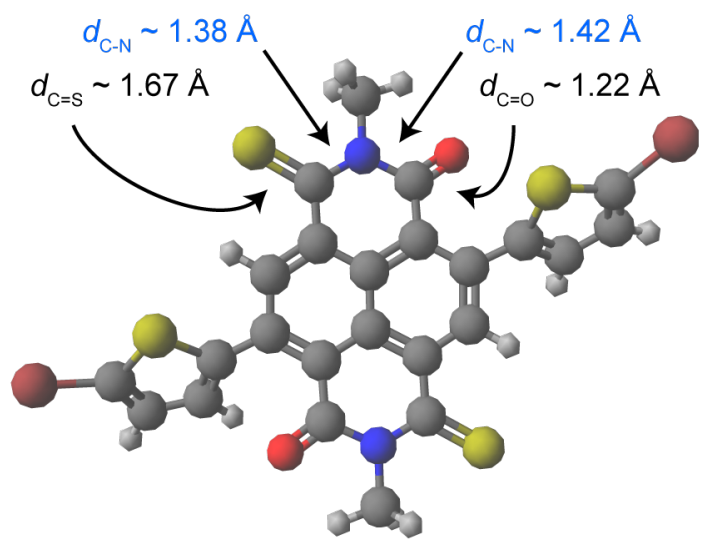

S2-Th

Figure S2. DFT-Calculated geometry optimizations (B3LYP functional, 6-31G(d) basis set) of oxygenated and thionated neutral monomers, indicating amide and thioamide $\mathrm{C}-\mathrm{N}$ (blue) and $\mathrm{C}=\mathrm{O} / \mathrm{C}=\mathrm{S}$ (black) bond lengths.
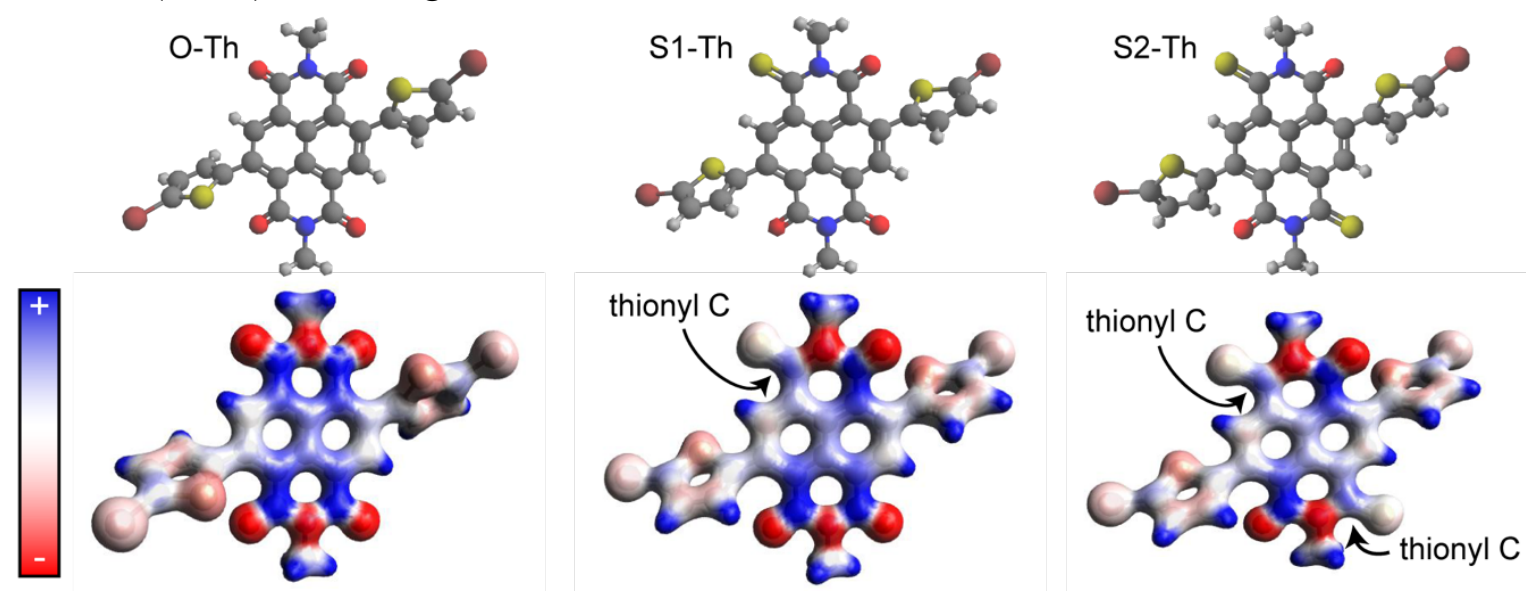

Figure S3. DFT-Calculated geometry optimizations (top) and electrostatic potential maps (bottom) of O-Th, S1-Th, and S2-Th. Thionyl carbons are more electron dense (less positive) than carbonyl carbons, which may be attributed to enhanced $\mathrm{N} \rightarrow \mathrm{C}$ charge transfer for thioamides. 
<smiles>[R]N1C(=O)c2c(-c3ccc(Br)s3)cc3c(=O)n([R])c(=S)c4cc(-c5ccc(Br)s5)c(c3c24)C(=S)N([R])C1=O</smiles>

Figure S4. Examples of possible resonance structures of the S2-Th anion-radical monomer, wherein unpaired electrons are localized to acceptor units and negative formal charges are assigned to electron withdrawing carbonyl oxygens (center) or thionyl sulfurs (right). Strong association between thionyl groups and nickel or palladium catalysts during anion-radical polymerization could lead to undesirable side reactions, including oxygen substitution of thionyl groups and catalyst poisoning.

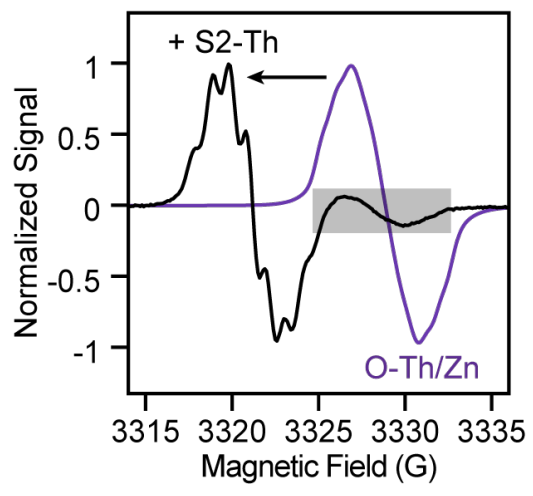

Figure S5. EPR spectra of O-Th/Zn before (purple) and after (black) addition of S2-Th. Arrow indicates the signal shift to lower magnetic field, attributed to $\mathrm{S} 2-\mathrm{Th} / \mathrm{Zn}$ formation. Grey box indicates residual O-Th/Zn signal, attributed to substoichiometric addition of S2-Th. 

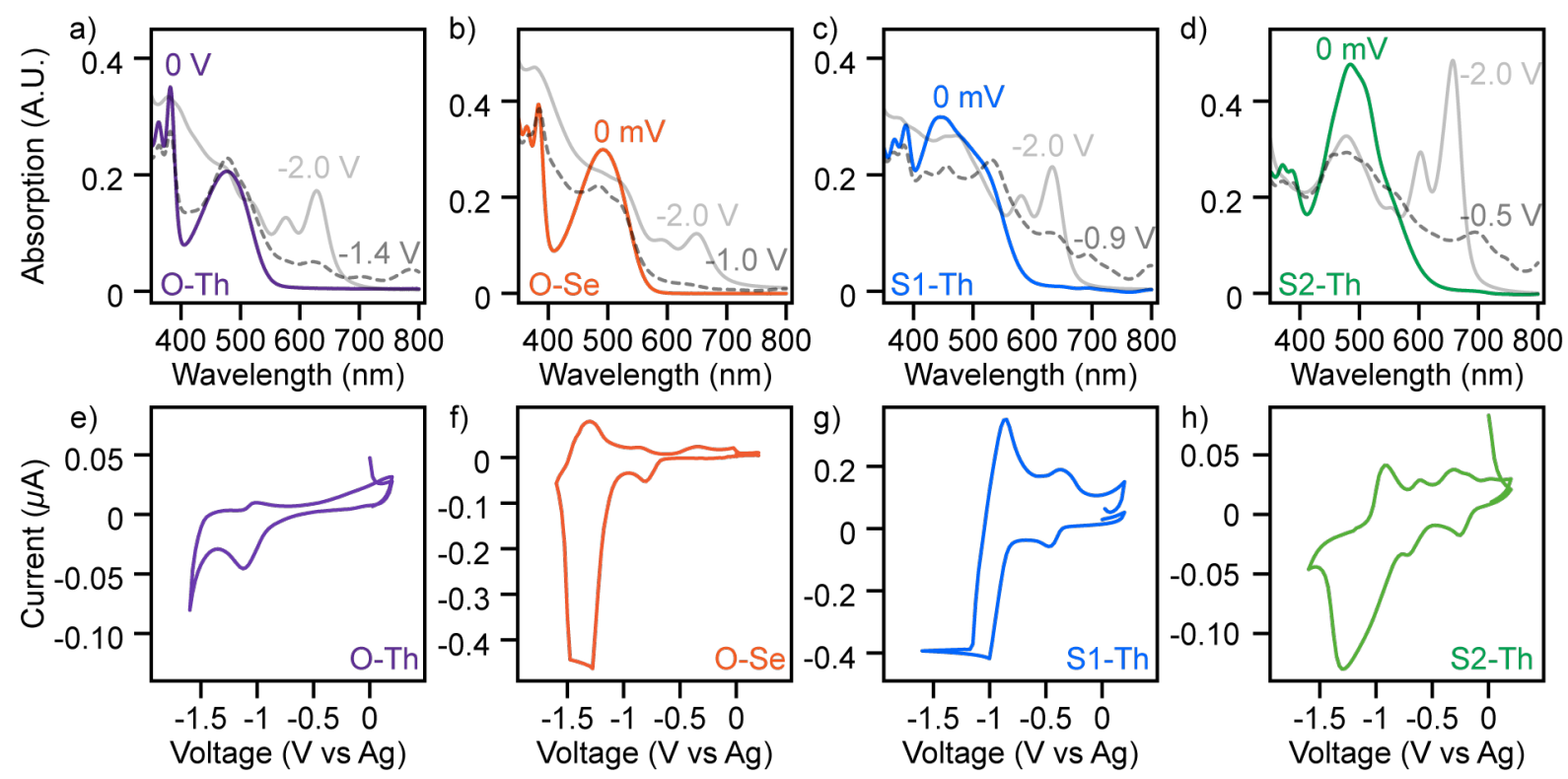

Figure S6. Spectroelectrochemistry studies of (a) O-Th, (b) O-Se, (c) S1-Th, and (d) S2-Th. Optical absorption spectra were obtained in tetrahydrofuran solution ( $2.5 \mathrm{mM}$ compound, $0.1 \mathrm{M}$ $\mathrm{TBAPF}_{6}$, and ambient atmosphere) at applied voltages corresponding to neutral, singly reduced, and doubly reduced states. Cyclic voltammetry measurements of (e) O-Th, (f) O-Se, (g) S1-Th, and (h) S2-Th were measured in situ relative to a silver pseudo reference electrode under ambient atmosphere to determine appropriate applied voltages.

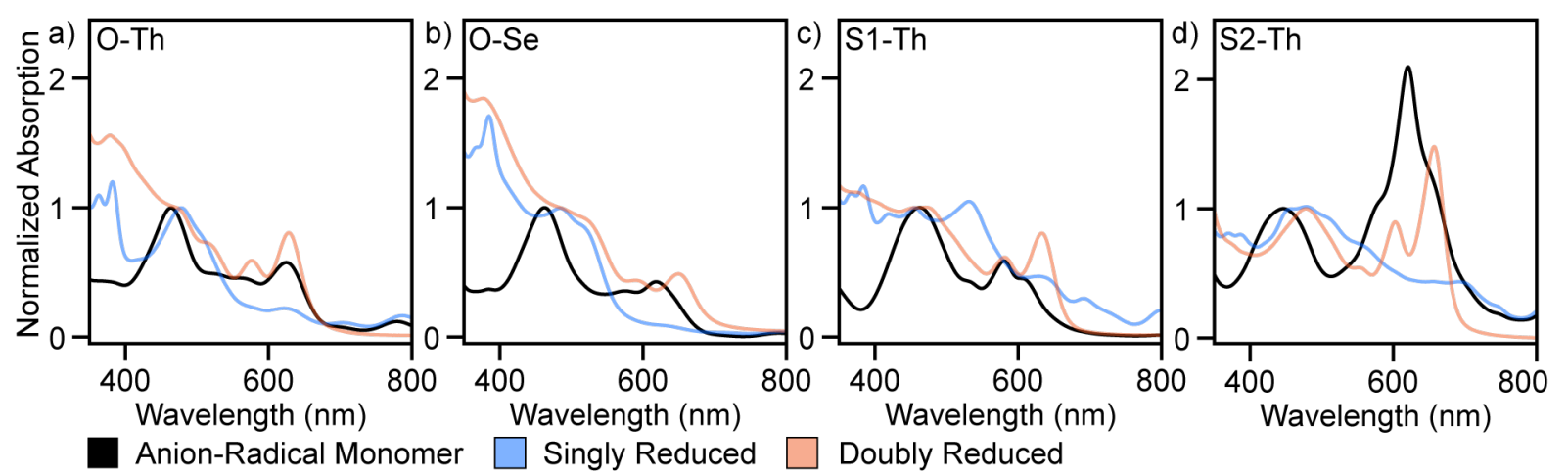

Figure S7. Normalized optical absorption spectra of anion-radical monomers compared to singly and doubly reduced states measured through spectroelectrochemical studies in Figure S4. Spectra are normalized to optical absorption bands with $\lambda_{\max } \sim 450 \mathrm{~nm}$, for comparison. 

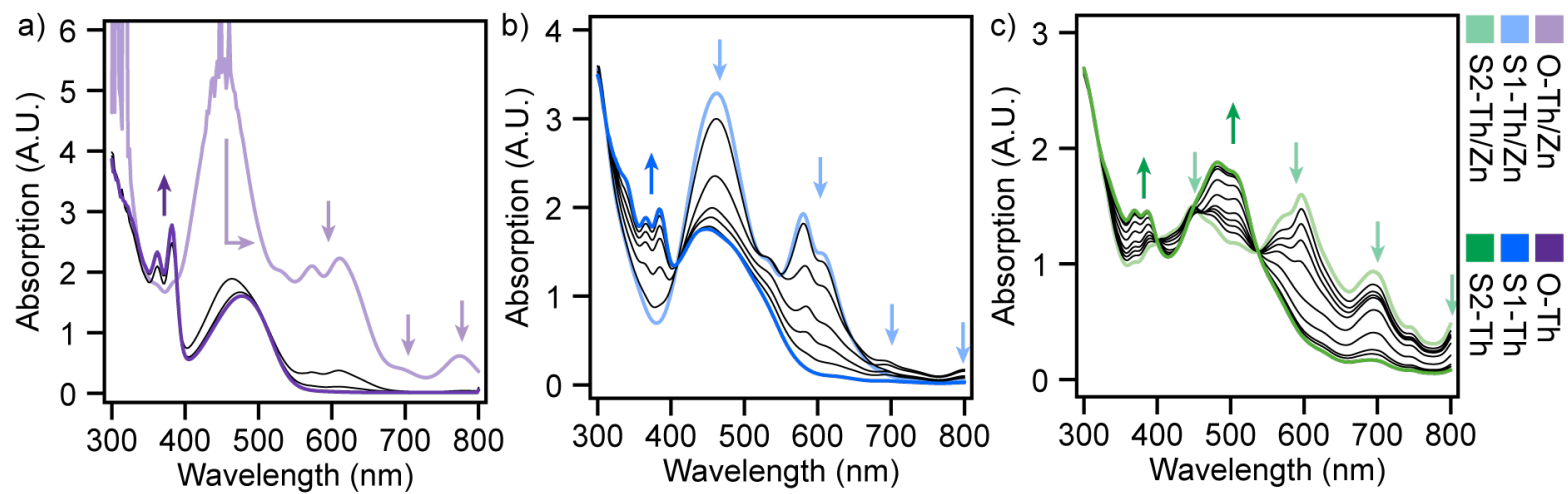

Figure S8. Time-resolved optical absorption spectra over one hour of (a) O-Th/Zn, (b) S1-Th/Zn, and (c) S2-Th/Zn following exposure to an ambient atmosphere to reform O-Th, S1-Th, and S2Th, respectively.

a)

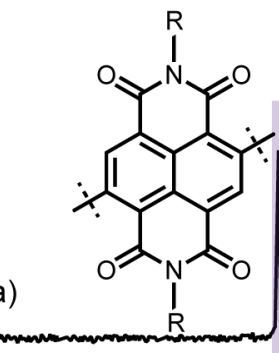
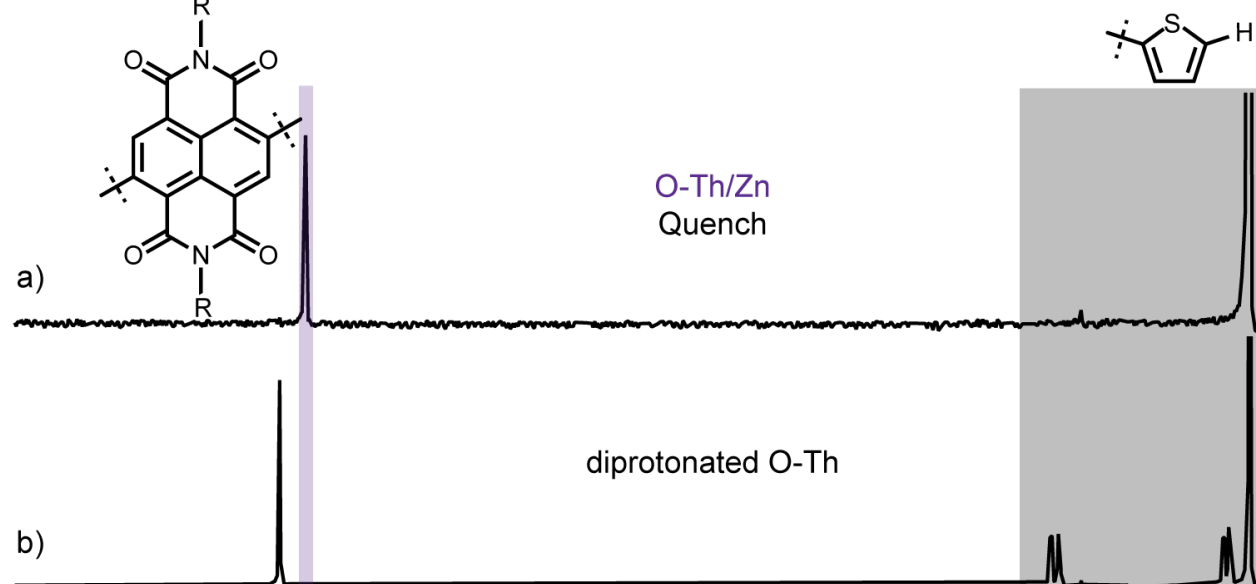

diprotonated O-Th

b)

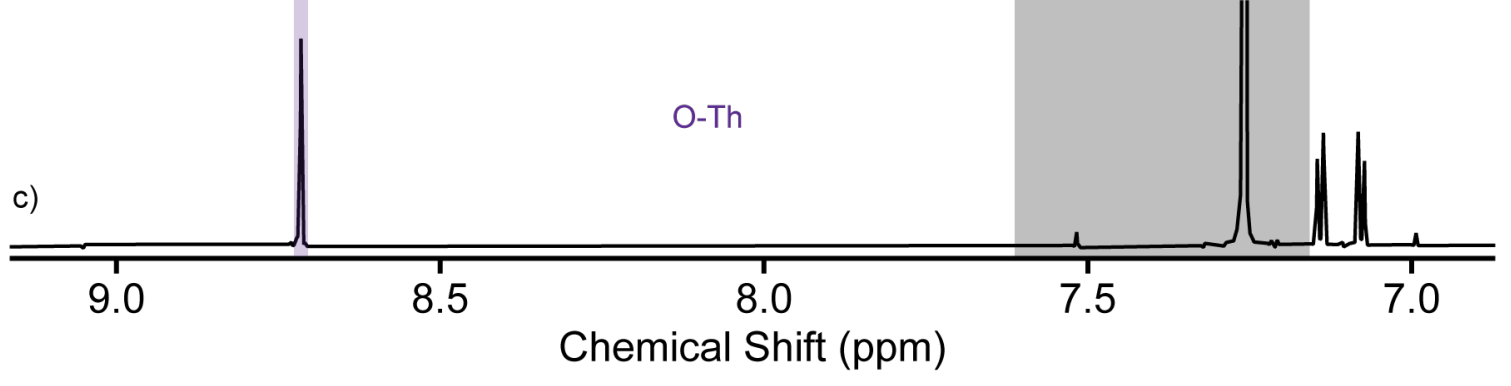

Figure S9. ${ }^{1} \mathrm{H}$ NMR analysis of (a) O-Th/Zn quenching product compared to (b) diprotonated O$\mathrm{Th}$, and (c) O-Th, indicating reformation of O-Th following exposure of O-Th/Zn to ambient atmosphere. 


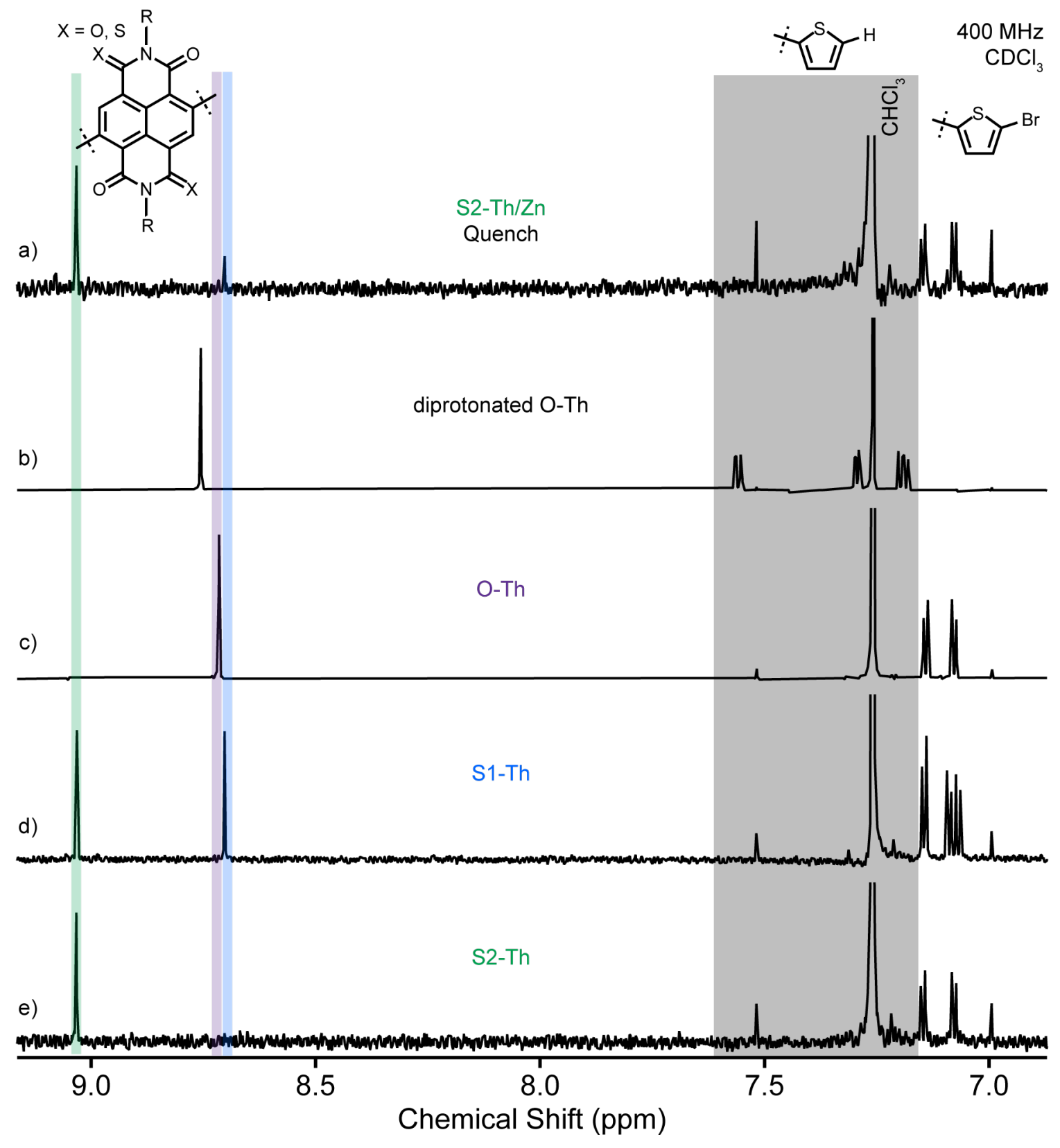

Figure S10. ${ }^{1} \mathrm{H}$ NMR analysis of (a) S2-Th/Zn quenching product compared to (b) diprotonated O-Th, (c) O-Th, (d) S1-Th, and (e) S2-Th, indicating reformation of S2-Th and partial oxidation to $\mathrm{S} 1-\mathrm{Th}$ and $\mathrm{P}$ following exposure of $\mathrm{S} 2-\mathrm{Th} / \mathrm{Zn}$ to an ambient atmosphere. 


\section{Anion-Radical Polymerization of Oxygenated and Thionated Monomers}

Table S2. Preliminary screening of $\mathrm{Ni}(\mathrm{dppe}) \mathrm{Cl}_{2}, \mathrm{Ni}(\mathrm{MesAn}) \mathrm{Br}_{2}$, and $\mathrm{Pd} / \mathrm{P}(t-\mathrm{Bu})_{3}$ anion-radical polymerization catalysts.

\begin{tabular}{|c|c|c|c|c|c|c|c|}
\hline No. & Monomer & Catalyst & \multicolumn{2}{|c|}{$[\mathrm{M}]:[\text { cat. }]^{\mathrm{a}}$} & $\mathrm{t}$ & $\begin{array}{c}M_{\mathrm{n}, \mathrm{GPC}}^{\mathrm{b}} \\
(\mathrm{kDa})\end{array}$ & $\bigoplus_{\mathrm{GPC}}{ }^{\mathrm{b}}$ \\
\hline 1 & \multirow{5}{*}{ O-Th } & \multirow{3}{*}{$\mathrm{Ni}($ dppe $) \mathrm{Cl}_{2}$} & $30: 1$ & $(31: 1)$ & 1 day & 10.6 & 2.00 \\
\hline 2 & & & $30: 1$ & $(31: 1)$ & 2 days & 6.2 & 1.54 \\
\hline 3 & & & $25: 1$ & $(25: 1)$ & 2 days & 3.5 & 2.26 \\
\hline 4 & & $\mathrm{Ni}(\mathrm{Mes} \mathrm{An}) \mathrm{Br}_{2}$ & $25: 1$ & $(25: 1)$ & 2 days & 3.4 & 1.09 \\
\hline $5^{\mathrm{c}}$ & & $\mathrm{Pd} / \mathrm{P}(t-\mathrm{Bu})_{3}$ & \multicolumn{2}{|c|}{ trace catalyst } & 1 day & \multicolumn{2}{|c|}{$\begin{array}{c}\text { polymer, residual } \\
\text { monomer }\end{array}$} \\
\hline 6 & \multirow{4}{*}{$\mathrm{O}-\mathrm{Se}$} & $\mathrm{Ni}($ dppe $) \mathrm{Cl}_{2}$ & $25: 1$ & $(25: 1)$ & 2 days & 6.3 & 1.49 \\
\hline 7 & & \multirow{2}{*}{$\mathrm{Ni}(\mathrm{Mes} \mathrm{An}) \mathrm{Br}_{2}$} & $30: 1$ & $(27: 1)$ & 1 day & 4.8 & 1.68 \\
\hline 8 & & & $25: 1$ & $(25: 1)$ & 2 days & 6.7 & 1.38 \\
\hline $9^{c}$ & & $\mathrm{Pd} / \mathrm{P}(t-\mathrm{Bu})_{3}$ & $\operatorname{trac}$ & talyst & 1 day & $\begin{array}{r}\text { poly } \\
\text { oligome } \\
\text { mon }\end{array}$ & $\begin{array}{l}\text { ner, } \\
\text { residual } \\
\text { ner }\end{array}$ \\
\hline $10^{\mathrm{c}}$ & \multirow{3}{*}{ S1-Th } & $\mathrm{Ni}($ dppe $) \mathrm{Cl}_{2}$ & \multicolumn{2}{|c|}{ trace catalyst } & 2 days & \multicolumn{2}{|c|}{ residual monomer } \\
\hline $11^{\mathrm{c}}$ & & $\mathrm{Ni}(\mathrm{Mes} \mathrm{An}) \mathrm{Br}_{2}$ & \multicolumn{2}{|c|}{ trace catalyst } & 1 day & \multicolumn{2}{|c|}{ residual monomer } \\
\hline $12^{\mathrm{c}}$ & & $\mathrm{Pd} / \mathrm{P}(t-\mathrm{Bu})_{3}$ & \multicolumn{2}{|c|}{ trace catalyst } & 2 days & \multicolumn{2}{|c|}{$\begin{array}{c}\text { oligomer, residual } \\
\text { monomer }\end{array}$} \\
\hline 13 & \multirow{3}{*}{$\mathrm{S} 2-\mathrm{Th}$} & $\mathrm{Ni}($ dppe $) \mathrm{Cl}_{2}$ & $30: 1$ & $(31: 1)$ & 1 day & \multicolumn{2}{|c|}{ residual monomer } \\
\hline $14^{\mathrm{c}}$ & & $\mathrm{Ni}(\mathrm{Mes} \mathrm{An}) \mathrm{Br}_{2}$ & \multicolumn{2}{|c|}{ trace catalyst } & 4 days & \multicolumn{2}{|c|}{ residual monomer } \\
\hline $15^{\mathrm{c}}$ & & $\mathrm{Pd} / \mathrm{P}(t-\mathrm{Bu})_{3}$ & \multicolumn{2}{|c|}{ trace catalyst } & 4 days & \multicolumn{2}{|c|}{$\begin{array}{l}\text { oligomer, residual } \\
\text { monomer }\end{array}$} \\
\hline
\end{tabular}

a Approximate values. Experimental molar ratios are provided in parentheses.

b 1,2,4-trichlorobenzene GPC measured at $140{ }^{\circ} \mathrm{C}$, calibrated against polystyrene standards.

${ }^{c}$ Using a pipette tip, a small amount of solid catalyst was added to the reaction flask prior to addition of the anion-radical monomer solution. These studies were only used for preliminary catalyst screening to determine whether or not any polymers $(>10 \mathrm{kDa})$, oligomers $(\sim 2-10 \mathrm{kDa})$, or residual monomeric species $(\sim 1 \mathrm{kDa})$ could be determined by GPC, NMR and/or optical absorption analysis. 
Table S3. Summary of anion-radical polymerization reaction conditions and properties.

\begin{tabular}{|c|c|c|c|c|c|c|c|c|}
\hline No. & Monomer & Catalyst & {$[\mathrm{M}]$} & [cat.]" & $\mathrm{t}$ & $\begin{array}{c}M_{\mathrm{n}, \mathrm{GPC}^{\mathrm{b}}} \\
(\mathrm{kDa})\end{array}$ & $\bigoplus_{\mathrm{GPC}^{\mathrm{b}}}$ & $\begin{array}{c}\% \\
\text { yield }\end{array}$ \\
\hline 1 & \multirow{8}{*}{ O-Th } & \multirow{8}{*}{$\mathrm{Ni}(\mathrm{dppp}) \mathrm{Cl}_{2}$} & 10:1 & $(11: 1)$ & $1 \mathrm{~h}$ & 3.0 & 1.17 & 31 \\
\hline 2 & & & $1: 4$ & $(1: 4)$ & $1 \mathrm{~h}$ & - & - & - \\
\hline 3 & & & $1: 12$ & $(1: 11)$ & $1 \mathrm{~h}$ & - & - & - \\
\hline 4 & & & $50: 1$ & $(52: 1)$ & $\begin{array}{c}10 \\
\text { min to } \\
5 \mathrm{~h}\end{array}$ & - & - & - \\
\hline 5 & & & 10:1 & $(10: 1)$ & $2.5 \mathrm{~h}$ & - & - & - \\
\hline 6 & & & $30: 1$ & $(32: 1)$ & $2.5 \mathrm{~h}$ & - & - & - \\
\hline 7 & & & $50: 1$ & $(48: 1)$ & $2.5 \mathrm{~h}$ & - & - & - \\
\hline 8 & & & 100:1 & $(95: 1)$ & $2.5 \mathrm{~h}$ & - & - & - \\
\hline 9 & \multirow{8}{*}{$\mathrm{O}-\mathrm{Se}$} & \multirow{8}{*}{$\mathrm{Ni}(\mathrm{dppp}) \mathrm{Cl}_{2}$} & 10:1 & $(10: 1)$ & $1 \mathrm{~h}$ & 6.7 & 1.73 & 67 \\
\hline 10 & & & $1: 4$ & $(1: 5)$ & $1 \mathrm{~h}$ & - & - & - \\
\hline 11 & & & $1: 12$ & $(1: 12)$ & $1 \mathrm{~h}$ & - & - & - \\
\hline 12 & & & $50: 1$ & $(48: 1)$ & $\begin{array}{c}10 \\
\min \text { to } \\
5 \mathrm{~h}\end{array}$ & - & - & - \\
\hline 13 & & & 10:1 & $(10: 1)$ & $2.5 \mathrm{~h}$ & 28.4 & 2.11 & 93 \\
\hline 14 & & & $30: 1$ & $(30: 1)$ & $2.5 \mathrm{~h}$ & 21.1 & 2.06 & 72 \\
\hline 15 & & & $50: 1$ & $(50: 1)$ & $2.5 \mathrm{~h}$ & 13.1 & 1.63 & 52 \\
\hline 16 & & & 100:1 & $(100: 1)$ & $2.5 \mathrm{~h}$ & 9.5 & 1.52 & 60 \\
\hline 17 & S1-Th & $\mathrm{Ni}(\mathrm{dppp}) \mathrm{Cl}_{2}$ & 10:1 & $(11: 1)$ & 1 day & - & - & 85 \\
\hline 18 & $\mathrm{~S} 2-\mathrm{Th}$ & $\mathrm{Ni}(\mathrm{dppp}) \mathrm{Cl}_{2}$ & 10:1 & $(11: 1)$ & 1 day & - & - & 77 \\
\hline
\end{tabular}

a Approximate values. Experimental molar ratios are provided in parentheses.

b $1,2,4$-trichlorobenzene GPC measured at $140^{\circ} \mathrm{C}$, calibrated against polystyrene standards. 

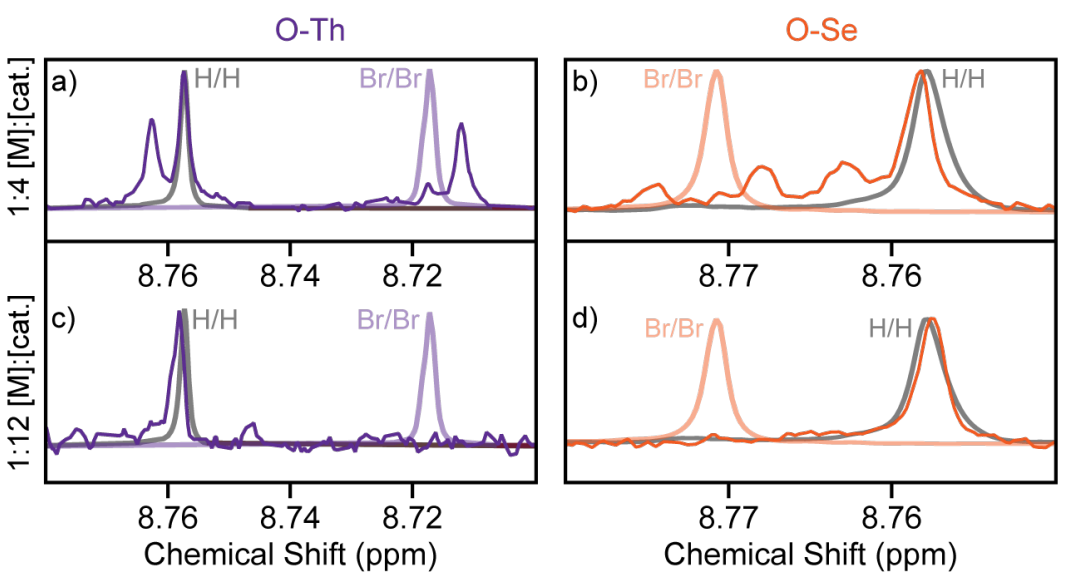

Figure S11. ${ }^{1} \mathrm{H}$ NMR (400 MHz, $\mathrm{CDCl}_{3}$ ) analysis of $\mathrm{O}-\mathrm{Th}$ and $\mathrm{O}$-Se following treatment with excess $\mathrm{Ni}(\mathrm{dppp}) \mathrm{Cl}_{2}$ for 1 hour, compared to diprotonated and dibrominated compounds.
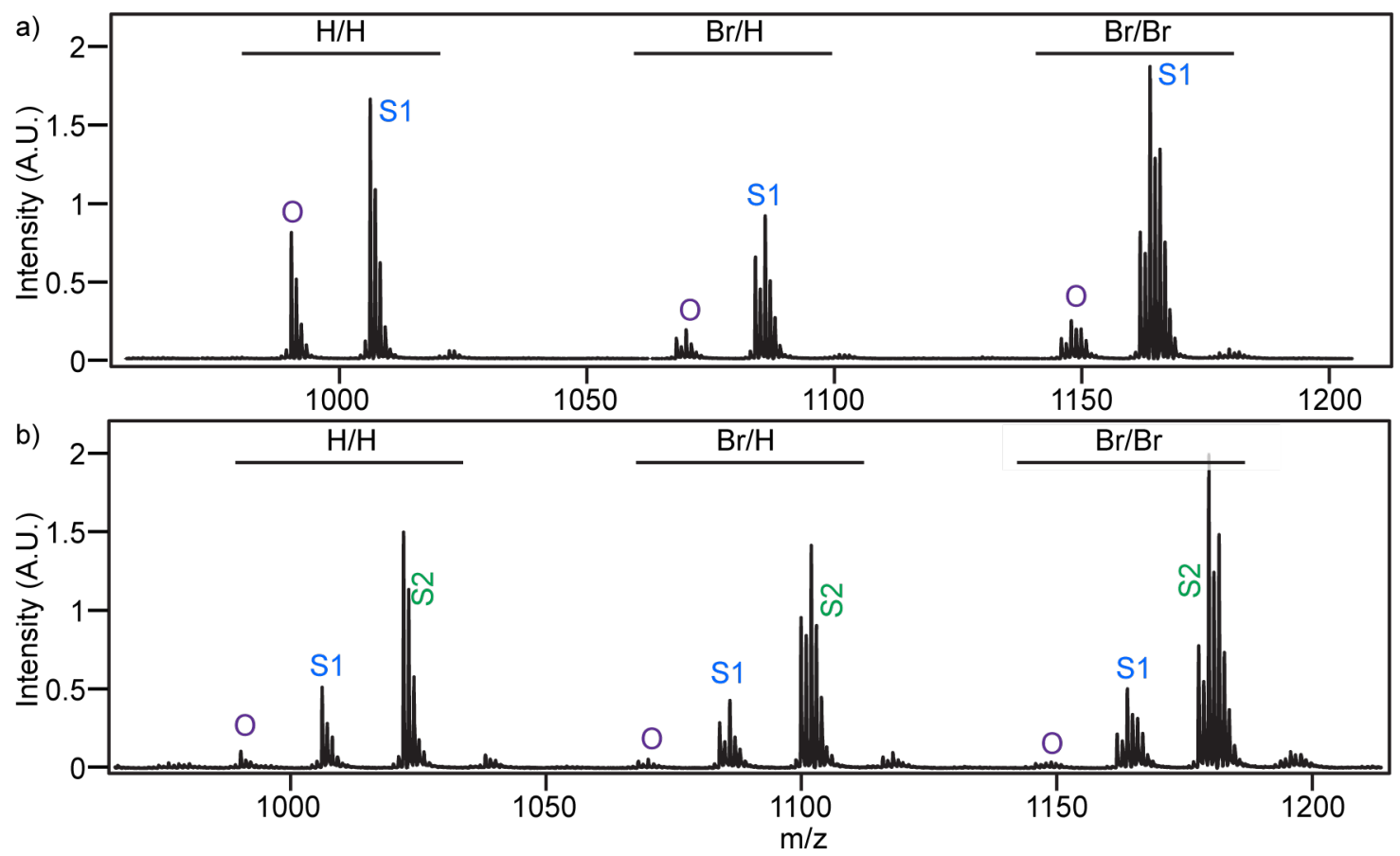

Figure S12. Mass spectra of (a) $\mathrm{S} 1-\mathrm{Th} / \mathrm{Zn}$ and (b) $\mathrm{S} 2-\mathrm{Th} / \mathrm{Zn}$ after $\mathrm{Ni}(\mathrm{dppp}) \mathrm{Cl}_{2}$ treatment, indicating partial protonation and oxygen-substitution. $\mathrm{S} 2=$ dithionated, $\mathrm{S} 1=$ monothionated, and $\mathrm{O}=$ fully oxygenated. 

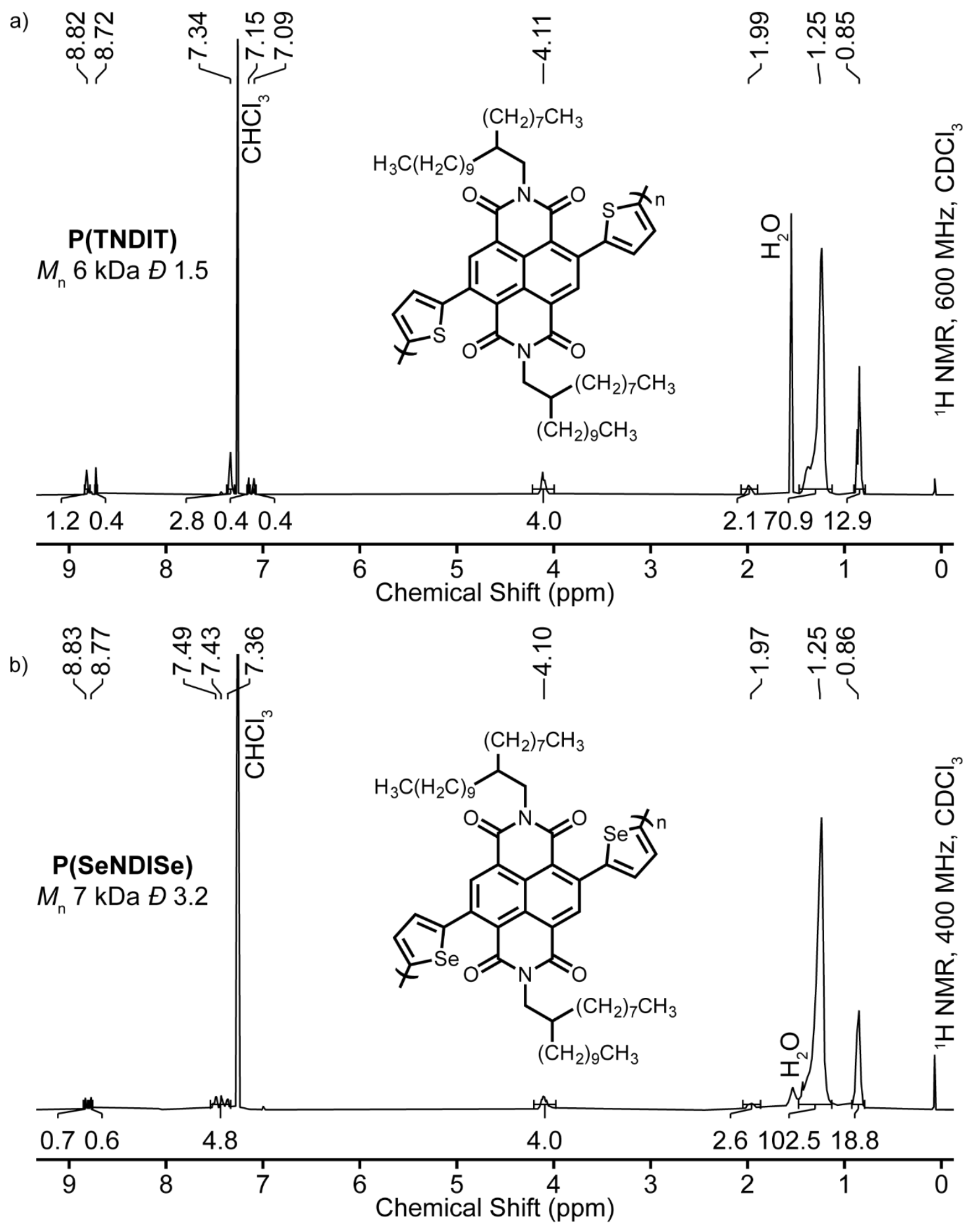

Figure S13. Representative ${ }^{1} \mathrm{H}$ NMR spectra of (a) P(TNDIT) and (b) P(SeNDISe). For reference to $\mathrm{P}(\mathrm{SeNDISe}), \mathrm{P}(\mathrm{TNDIT})$ with larger $M_{\mathrm{n}}$ was synthesized through anion-radical polymerization using $\mathrm{Ni}(\mathrm{dppe}) \mathrm{Cl}_{2}$. 


\section{Supplementary Computational Calculations}
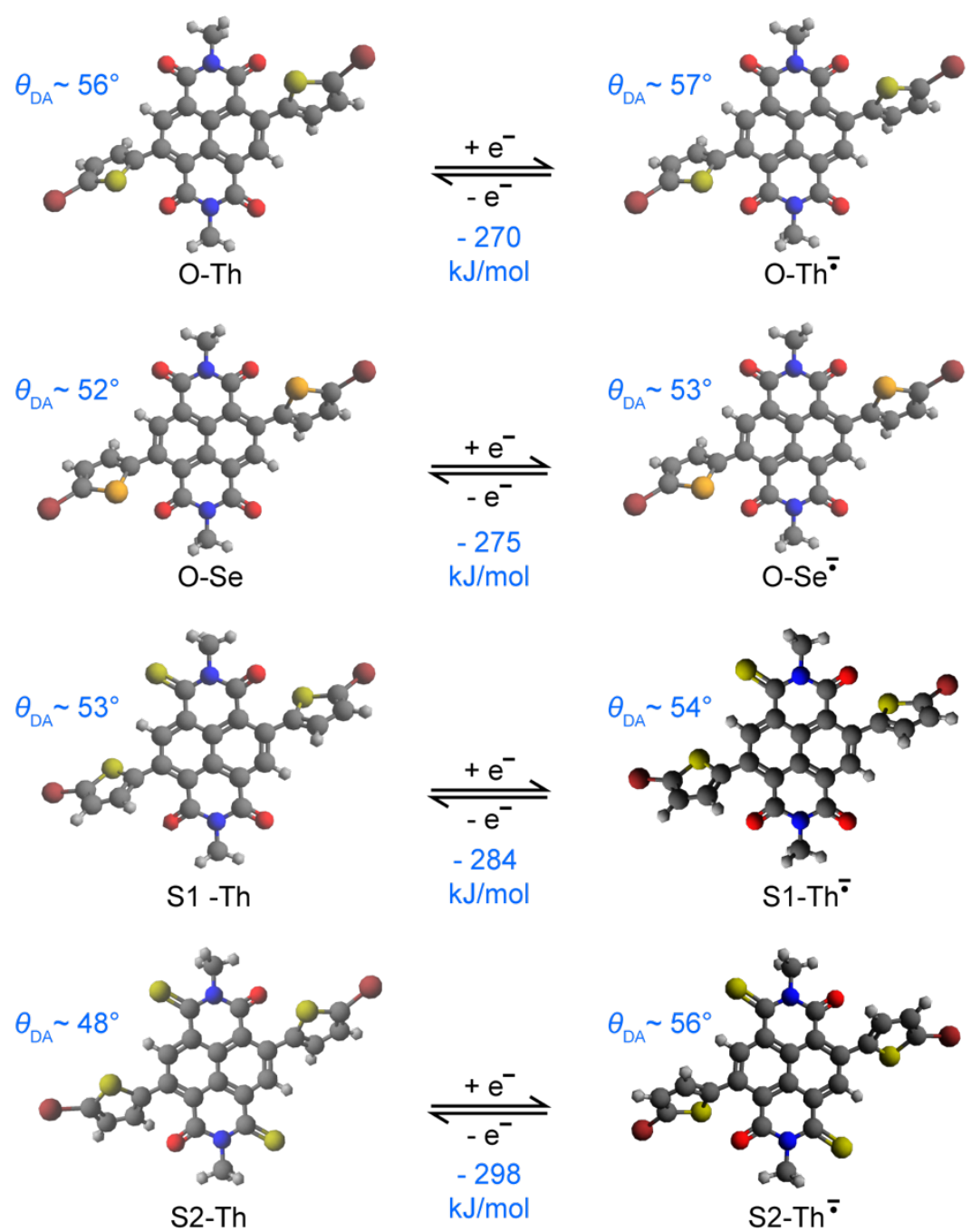

Figure S14. DFT-calculated geometry optimizations and single point energy calculations of neutral and reduced O-Th, O-Se, S1-Th, and S2-Th, indicating dihedral angles $\left(\theta_{\mathrm{DA}}\right)$ between NDI and $\mathrm{Th} / \mathrm{Se}$ units (B3LYP functional and 6-31G(d) basis set) and relative energies between neutral and reduced monomers (B3LYP functional and 6-311+G(d,p) basis set). 